\title{
Gastrinoma hepático primario, presentación de caso extremadamente raro y su resolución quirúrgica, en un hospital de tercer nivel en México
}

\author{
Primary hepatic gastrinoma, extremely rare case presentation and its surgical resolution, \\ in a third level hospital in Mexico
}

Rogelio González-López¹, Jonatan Ramírez-Castañeda', José A. Ortega-Jiménez*, Eugenio García-Cano², Mirza Palacios-Rojo ${ }^{3}$, Roberto A. Núñez-González', Antonio S. Sosa-Luis ${ }^{1}$ y Claudia T. Gutiérrez-Quiroz

${ }^{1}$ Servicio de Cirugía General, Unidad Médica de Alta Especialidad, Instituto Mexicano del Seguro Social (IMSS), Puebla, Puebla; ${ }^{2}$ Servicio de Cirugía General, Cirugía Plástica y Reconstructiva, Hospital Betania Christus Muguerza, Puebla, Puebla; ${ }^{3}$ Servicio de Medicina Interna, Hospital Regional de Río Blanco, Servicios de Salud de Veracruz, Río Blanco, Veracruz; ${ }^{4}$ Servicio de Anatomía Patológica, Unidad Médica de Alta Especialidad, IMSS, Puebla, Puebla. México

\begin{abstract}
Resumen
Los gastrinomas son neoplasias neuroendocrinas funcionales raras, y la mayoría se localizan en el duodeno (70-90\%) o en el páncreas (2-30\%), pero también existen otras localizaciones ectópicas poco comunes. El gastrinoma hepático primario es extremadamente raro, con menos de 40 casos reportados en la literatura médica. Su baja incidencia y su presentación clínica inespecífica lo convierten en una enfermedad difícil de diagnosticar. Al realizar un diagnóstico oportuno puede ser tratado mediante resección quirúrgica con altas posibilidades de éxito. El objetivo de este trabajo es presentar un caso clínico de gastrinoma hepático primario en México, tratado de manera exitosa mediante segmentectomía hepática derecha.
\end{abstract}

Palabras clave: Gastrinoma hepático primario. Tratamiento quirúrgico. Segmentectomía.

\begin{abstract}
The gastrinomas are rare functional neuroendocrine neoplasms, most are localized to the duodenum (70-90\%) or the pancreas $(2-30 \%)$, but less common ectopic sites have been reported. The primary hepatic gastrinoma is extremely rare, with less tan 40 cases reported in the medical literature. Its low incidence and its non specific clinical presentation make it a difficult disease to diagnose. Providing a timely diagnosis the patient can be treated by surgical resection with high chances of success. The objective of this paper is to describe a case of primary hepatic gastrinoma in Mexico, successfully treated by right liver segmentectomy.
\end{abstract}

Keywords: Primary hepatic gastrinoma. Surgical treatment. Segmentectomy.

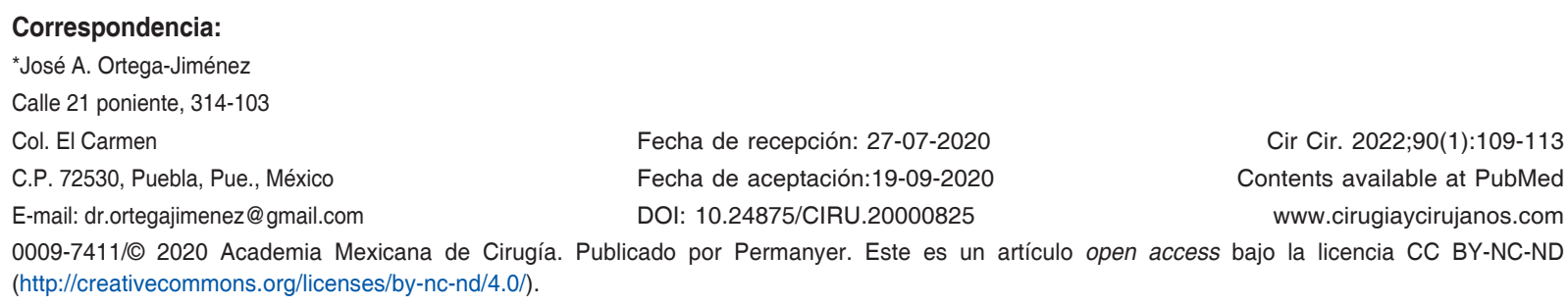




\section{Introducción}

El síndrome Zollinger-Ellison fue descrito por primera vez en el año 1955 por dos cirujanos, R.M. Zollinger y E.H. Ellison'. Es la segunda neoplasia neuroendocrina funcional más común, y también es considerado como uno de los tumores neuroendocrinos (TNE) más difíciles de tratar². Se debe a la hipersecreción de gastrina producida por un tumor llamado gastrinoma ${ }^{3}$.

Los gastrinomas son neoplasias neuroendocrinas funcionales raras, con una incidencia anual en la mayoría de las áreas geográficas de aproximadamente 0,5-1/1.000.000 habitantes/año. La incidencia es mayor en los hombres que en las mujeres y la edad media de presentación se encuentra entre los 45 y 50 años. A pesar de que estos tumores son de crecimiento lento, en el $60-70 \%$ de los casos son malignos y un $25 \%$ tienen un crecimiento rápido ${ }^{4}$. Pueden presentarse de manera esporádica $(75 \%)$ o formando parte del síndrome de neoplasia endocrina múltiple tipo $1(25 \%)^{5}$.

El gastrinoma hepático primario es una enfermedad rara, con menos de 40 casos reportados en la literatura médica, y debido a que se localiza en un órgano en donde las metástasis son comunes, el diagnóstico se vuelve todo un reto ${ }^{6}$.

Se presenta un caso clínico de gastrinoma hepático primario tratado mediante resección quirúrgica en un hospital del tercer nivel en México, con resultados satisfactorios inmediatos y a 8 meses de seguimiento.

\section{Caso clínico}

Varón de 50 años sin antecedentes familiares o personales patológicos de importancia, que inicia su padecimiento con epigastralgia y pirosis de 2 años de evolución, y a los 6 meses de iniciado el cuadro se añaden diarrea crónica y emesis ocasional. Además, ha presentado pérdida de peso de más de $10 \mathrm{~kg}$ en un lapso de 12 meses.

En la exploración física inicial, únicamente destaca dolor a la palpación en el epigastrio y el hipocondrio derecho, sin lesiones ni visceromegalias identificables a la palpación, sin datos de irritación peritoneal, y el resto sin agregados importantes de comentar.

Durante su protocolo de estudio fue sometido a cuatro panendoscopias realizadas por diferentes operadores y en distintas instituciones, todas con reportes histopatológicos (RHP). Los hallazgos encontrados y sus respectivos RHP, fueron los siguientes:
1) Hallazgos sugestivos de candidiasis esofágica, bulboduodenitis erosiva, gastritis crónica. RHP: biopsia de esófago con mucosa de Barret sin displasia, mucosa cardial sin displasia.

2) Esofagitis grado II, gastritis superficial, duodeno normal. RHP: biopsia de esófago con esofagitis crónica.

3) Moniliasis esofágica, gastritis y duodenitis mínimas. RHP: biopsia de esófago con esofagitis crónica, biopsia gástrica con gastritis crónica leve, no asociada a Helicobacter pylori.

4) Gastroduodenitis crónica. RHP: biopsia gástrica con gastritis no atrófica, sin metaplasia o diplasia, sin evidencia de células malignas en el tejido estudiado. Se realiza también colonoscopia, encontrándose imagen compatible con colon espástico.

Se decide realizar una tomografía computada (TC) simple y con doble contraste, encontrando engrosamiento en el tercio distal del esófago y en la unión gastroesofágica. En el segmento VII del hígado se observa una lesión nodular de $49 \mathrm{~mm}$, de bordes regulares, definidos, homogéneos, con densidad en fase simple de 15-35 UH (Fig. 1), y el resto del estudio sin alteraciones. Debido a los resultados de la TC, se realiza biopsia percutánea del tumor hepático (Fig. 2), que reporta tumor neuroendocrino moderadamente diferenciado de grado 2, CK7 negativo, CK20 negativo, CEA negativo, HEP-PAR1 negativo, sinaptofisina positivo, cromogranina A positivo y KI67 positivo (5\%).

También se lleva a cabo un centellograma con octreotida-Tc-99m que muestra una lesión hipercaptante en el lóbulo derecho del hígado (Fig. 3). No se encuentran áreas anormales en el resto del cuerpo entero rastreado. Se complementa el protocolo con tomografía computada por emisión de positrones (PET) de cuerpo entero, encontrando una lesión hepática en el segmento VI-VII, hipodensa, con aumento de metabolismo (Fig. 4), y aumento difuso de metabolismo en el esófago y el cuerpo gástrico.

Con el resultado obtenido de NET se realizan pruebas séricas de ácido 5-hidroxindolacético, cromogranina A y gastrina, con resultados de $2.2 \mathrm{mg} / 24 \mathrm{~h}$, $33.9 \mathrm{nmol} / \mathrm{l}$ y $7715 \mathrm{pg} / \mathrm{ml}$, respectivamente, confirmando el diagnóstico de gastrinoma hepático primario.

El paciente es sometido a cirugía, encontrando un hígado de tamaño normal, con presencia de tumor en el segmento VII, de aproximadamente $7 \mathrm{~cm}$ de diámetro; el resto de los segmentos sin alteraciones. Se realiza segmentectomía derecha posterior (segmentos VI y VII). El paciente presentó adecuada evolución y se egresó al cuarto día posquirúrgico. 


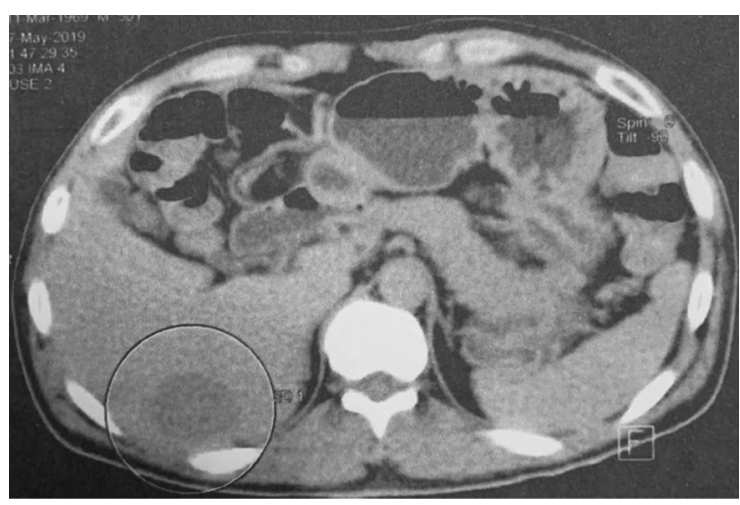

Figura 1. Tomografía computarizada simple de abdomen, corte axial. Se observa una lesión nodular hepática en el segmento VII, hipodensa, de bordes regulares y definidos.

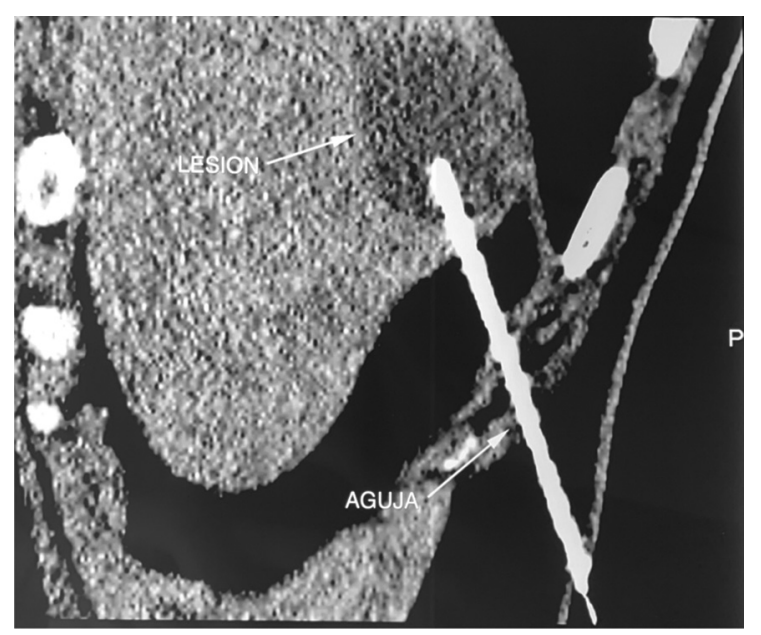

Figura 2. Biopsia percutánea. Las flechas señalan la lesión hepática circunscrita y la aguja utilizada para la toma de biopsia.

El RHP de la pieza quirúrgica reporto NET de grado intermedio (G2) de $6.4 \mathrm{~cm}$, ampliación de margen quirúrgico libre de neoplasia, sinaptofisina positivo, cromogranina positivo, CD56 positivo, KI67 positivo (5-7\%), P53 positivo y citoqueratinas positivo (Fig. 5).

Actualmente, a 8 meses de la intervención, el paciente se refiere asintomático. Cuenta con ultrasonido y TC abdominal sin alteraciones, y controles séricos de gastrina dentro de los parámetros normales $(<115 \mathrm{pg} / \mathrm{ml})$.

\section{Discusión}

Los gastrinomas son neoplasias raras que se localizan habitualmente en "el triángulo del gastrinoma», una región anatómica imaginaria, delimitada en su

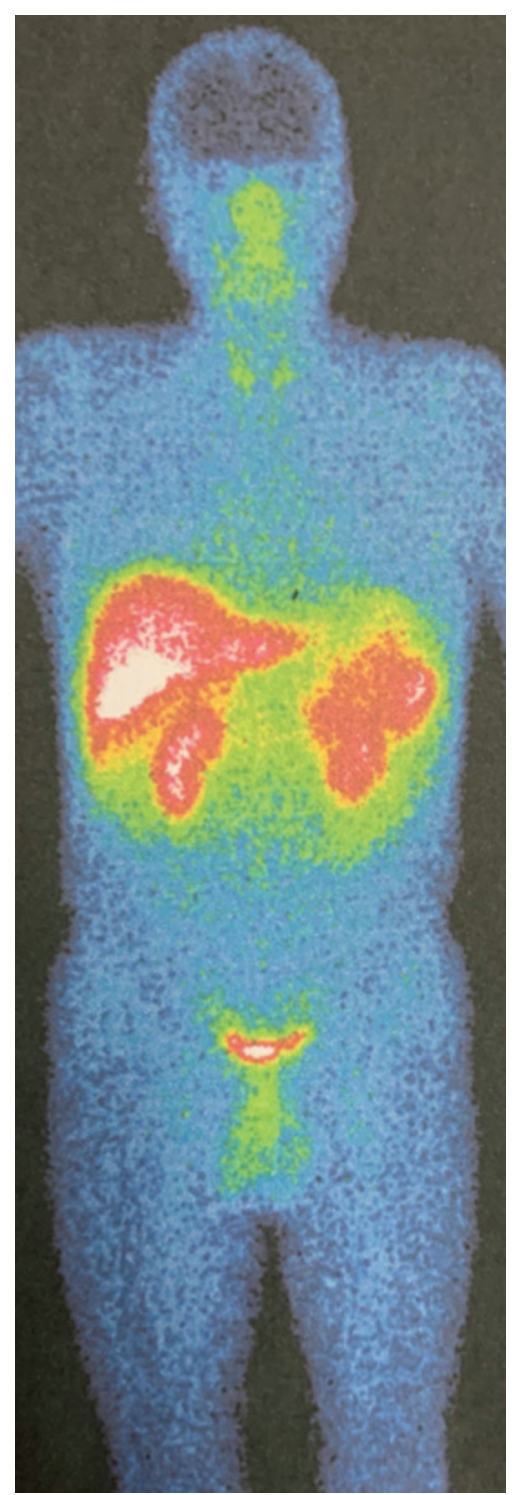

Figura 3. Gammagrafía. Corte coronal que demuestra un aumento de la captación en el lóbulo hepático derecho.

porción superior por la confluencia del conducto cístico y el conducto hepático, medialmente por la unión del cuello y la cabeza del páncreas, e inferiormente por la unión de la segunda y la tercera porciones del duodeno ${ }^{7}$. La mayoría de los gastrinomas se localizan en el duodeno (70-90\%) o en el páncreas (2-30\%), pero también existen otras localizaciones ectópicas raras, como el ovario, el estómago, los ganglios linfáticos, el corazón, el hígado y los conductos biliares². Se ha reportado que solo un $5.6 \%$ se encuentran de manera extrapancreática, extraduodenal o extranodal $^{8}$. Debido a que el hígado es un órgano en el que los NET suelen presentar metástasis con frecuencia, 


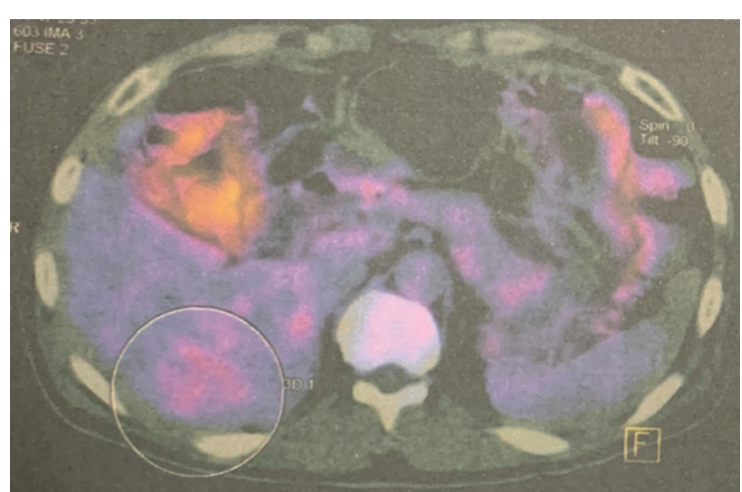

Figura 4. Tomografía por emisión de positrones. Se observa un aumento del metabolismo en la lesión localizada en el lóbulo hepático derecho.

y a que solo se encuentran reportados en la literatura médica menos de 40 casos de gastrinoma hepático primario ${ }^{6}$, se debe realizar una búsqueda exhaustiva para descartar tumores en otros sitios. Por esta razón, en este paciente se realizaron diversos estudios diagnósticos que descartaron una segunda lesión maligna, eliminando en primera instancia la posibilidad de una neoplasia en la unión gastroesofágica por el engrosamiento encontrado en la TC.

Las manifestaciones clínicas de este tumor están asociadas a la hipersecreción de gastrina, produciéndose una elevación de ácido gástrico, que es la causa de la aparición del síndrome de Zollinger-Ellison, caracterizado por enfermedad ácido-péptica y alteración de la motilidad gastrointestinal ${ }^{9}$. La presentación clínica clásica de este síndrome es con dolor abdominal (75\%) y diarrea crónica $(73 \%)^{6}$, como ocurrió en nuestro paciente.

En algunos trabajos se ha reportado que la gammagrafía/centellografía resulta ser más sensible que cualquier otro método de imagen para la identificación de un gastrinoma ${ }^{10}$. Localiza los NET de manera exitosa hasta en un $78-86 \%$ y se ha convertido en el estudio de imagen de elección para los pacientes con sospecha de gastrinoma ${ }^{7}$. Por otra parte, la PET ha revolucionado en los últimos años los protocolos de estudio para buscar neoplasias, gracias a su capacidad de visualizar actividad metabólica. Ambos estudios fueron aplicados en este paciente y sus resultados corroboraron que se trataba de un NET hepático primario, descartando de manera contundente una posible lesión secundaria en otro órgano. Posterior a la realización de pruebas séricas y al encontrar unos valores de gastrina extremadamente altos, se concluyó el diagnóstico de gastrinoma hepático primario.

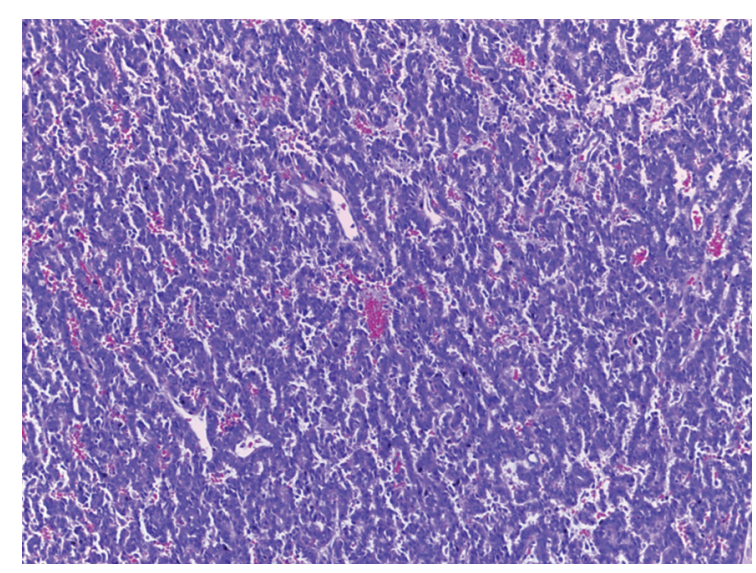

Figura 5. Microfotografía con acercamiento de la lesión, en la que se observan trabéculas y pseudoglándulas, con presencia de núcleos homogéneos y citoplasma eosinófilo.

La importancia de un diagnóstico y un tratamiento oportunos radica en que el $65 \%$ de los gastrinomas presentan un curso maligno ${ }^{7}$, y además la sintomatología presentada por los pacientes afecta su calidad de vida de manera significativa.

La hipersecreción gástrica del síndrome de Zollinger-Ellison puede manejarse de manera general con inhibidores de la bomba de protones o con fármacos antisecretores; sin embargo, la cirugía es el único tratamiento curativo para los gastrinomas ${ }^{5}$. A pesar de los pocos casos reportados de gastrinoma hepático primario, la resección quirúrgica debe ser el tratamiento de elección. La cirugía es la única posibilidad de cura, con tasas de éxito de hasta el $86 \%$, observándose unos valores de gastrina normales en el posoperatorio temprano en el $60 \%$ de los casos. La supervivencia a 15 años en los pacientes operados es del $98 \%$, frente al $74 \%$ en los no operados ${ }^{6}$.

En este paciente se decidió realizar resección completa del tumor con segmentectomía hepática derecha posterior, obteniendo márgenes libres de neoplasia. Esto provee al paciente un tratamiento curativo, aumentando su supervivencia a largo plazo y mejorando su calidad de vida.

\section{Conclusiones}

El gastrinoma hepático primario es un tumor sumamente raro, con muy pocos casos reportados en el mundo. El dolor abdominal, la diarrea, el vómito y la pérdida de peso son algunos de los síntomas que merman la vida diaria de los pacientes. Para llegar a su diagnóstico se requiere contar con un estudio de 
imagen que evidencie la lesión tumoral hepática, toma de biopsia de dicha lesión con RHP compatible con NET, realización de estudios de extensión que descarten lesiones neoplásicas en otros órganos y demostrar unos valores séricos elevados de gastrina. Por tratarse de un tumor con altas posibilidades de presentar un curso maligno, la resección quirúrgica es la prioridad.

Este caso clínico nos muestra que con un tratamiento adecuado y oportuno se pueden aumentar la calidad y la expectativa de vida de estos pacientes.

\section{Financiamiento}

Los autores declaran no haber recibido financiamiento para este estudio.

\section{Conflicto de intereses}

Los autores declaran no tener conflicto de intereses.

\section{Responsabilidades éticas}

Protección de personas y animales. Los autores declaran que para esta investigación no se han realizado experimentos en seres humanos ni en animales.
Confidencialidad de los datos. Los autores declaran que han seguido los protocolos de su centro de trabajo sobre la publicación de datos de pacientes.

Derecho a la privacidad y consentimiento informado. Los autores han obtenido el consentimiento informado de los pacientes y/o sujetos referidos en el artículo. Este documento obra en poder del autor de correspondencia.

\section{Bibliografía}

1. Norton JA, Foster DS, Ito T, Jensen RT. Gastrinomas: medical or surgical treatment. Endocrinol Metab Clin North Am. 2018;47:577-601.

2. Norton JA, Foster DS, Blumgart LH, Poultsides GA, Visser BC, Fraker DL. Incidence and prognosis of primary gastrinomas in the hepatobiliary tract. JAMA Surg. 2018;153:e175083

3. Denève $\mathrm{E}$, Hamoui M, Chalancon A, Blanc PM, Aufort S, Fabre JM, et al. A case of intrahepatic gastrinoma. Ann Endocrinol (Paris). 2009;70:242-5.

4. Fernández-Cruz L, Pelegrina A. Cirugía del gastrinoma: resultados inmediatos y a largo plazo. Cir Esp. 2015;93:390-5.

5. Atema JJ, Amri R, Busch ORC, Rauws EAJ, Gouma DJ, Nieveen van Dijkum EJM. Surgical treatment of gastrinomas: a single-centre experience. HPB (Oxford). 2012;14:833-8.

6. Pipek LZ, Jardim YJ, de Mesquita GHA, Nii F, de Almeida Medeiros KA, Carvalho BJ. Large primary hepatic gastrinoma in young patient treated with trisegmentectomy: a case report and review of the literature. World J Hepatol. 2018;10:517-22.

7. Tsalis K, Vrakas G, Vradelis S, Dimoulas A, Pilavaki M, Papaemmanouil S, et al. Primary hepatic gastrinoma: report of a case and review of literature. World J Gastrointest Pathophysiol. 2011;2:26-30.

8. Wu PC, Alexander HR, Bartlett DL, Doppman JL, Fraker DL, Norton JA. A prospective analysis of the frequency, location, and curability of ectopic (nonpancreaticoduodenal, nonnodal) gastrinoma. Surgery. 1997;122:1176-82.

9. Zollinger RM, Ellison EH. Primary peptic ulcerations of the jejunum associated with islet cell tumors of the pancreas. Ann Surg. 1955;142:709-28.

10. Arques PB, Benito IH, Navarro AM. Gammagrafía con ${ }^{111} \mathrm{In}$-octreotide en un caso de gastrinoma hepático primario. Rev Esp Med Nucl. 2001;20:381-5. 